\title{
Visualization and Quantification of the Cerebral Microcirculation using Contrast Enhanced Ultrasound Particle Tracking Velocimetry
}

\author{
Z. Zhang', M. Hwang' ${ }^{2}$ T. J. Kilbaugh ${ }^{3}$, A. Sridharan ${ }^{2}$, J. Katz ${ }^{*}$ \\ ${ }^{1}$ Johns Hopkins University, Department of Mechanical Engineering, Baltimore, USA. \\ ${ }^{2}$ Children's Hospital of Philadelphia, Department of Radiology, Philadelphia, USA. \\ ${ }^{3}$ Children's Hospital of Philadelphia, Department of Anesthesiology and Critical Care Medicine, \\ Philadelphia, USA. \\ *katz@jhu.edu
}

\begin{abstract}
Noninvasive measurements of the regional microvascular perfusion might lead to sensitive biomarkers for the changes in intracranial hemodynamics that could guide timely surgical interventions for neonatal brain injuries. The current work utilizes a clinically available contrast enhanced ultrasound (CEUS) system and particle tracking velocimetry to perform ultrasound localization microscopy for measuring the microcirculation in piglets. A new deep learning method based on U-net is proposed for enhancing noisy raw CEUS images and detecting the microbubbles. Subsequently, the bubbles are tracked using a Kalman filter based method, which incorporates conditions of spatio-temporal consistency in flow direction and globally optimizes the assignment of bubbles to trajectories. Based on analysis of synthetic data, the U-net results demonstrate significant improvement in the processing speed and localization accuracy over a conventional blind deconvolution method. Visualization of the microvasculature is performed by superposing the bubble trajectories, enabling depiction of a complex micro-vessel network, where neighboring vessels separated by $40 \mu \mathrm{m}$ can be distinguished. The corresponding perfusion map shows the velocity distribution in these vessels. Based on the current frame rate ( $44 \mathrm{fps}$ ), speeds in the 0.1 to $12 \mathrm{~cm} / \mathrm{s}$ range can be well captured. These methods show promise as potential clinical tools for bedside measurement of cerebral microcirculation.
\end{abstract}

\section{Introduction}

Noninvasive measurements of the cerebral blood flow (CBF) provide vital information about the hemodynamic conditions that could guide timely surgical interventions for various types of neonatal brain injuries. Conventionally, Magnetic Resonance Imaging (Leliefeld et al. 2008) or Computed Tomography (Dankbaar et al. 2010) are used for mapping the regional distributions of CBF. Due to their millimetric scale spatial resolutions, these techniques cannot distinguish the macrovascular perfusions from the microperfusions (Demené et al. 2021). Flow parameters measured in major cerebral arteries are inadequate for monitoring the complex intracranial dynamic responses under varying hemodynamic conditions (Hanlo et al. 1995). In contrast, the microvascular flow in different cerebral regions is sensitive to hemodynamics changes (Zaharchuk et al. 1999). Therefore, techniques that enable measurements of regional microperfusion might lead to more sensitive markers for various cerebral pathological conditions.

As a convenient bedside tool, contrast-enhanced ultrasound (CEUS) imaging has been introduced for 
$14^{\text {th }}$ International Symposium on Particle Image Velocimetry -- ISPIV 2021

August 1--5, 2021

evaluating the cerebral perfusion in neonatal brains (Hwang 2019). CEUS utilizes intravascular microbubbles $(<5 \mu \mathrm{m}$ in diameter) to visualize the blood flow. Several postprocessing techniques have been developed to quantify the flow parameters based on CEUS images, including ultrasound imaging velocimetry or echo-PIV (Crapper et al. 2000; Kim et al. 2004; Poelma 2017; Zhang et al. 2020), and echoparticle tracking velocimetry (Sampath et al. 2018; Jeronimo et al. 2020), which is also referred to as Ultrasound Localization Microscopy (ULM) in the CEUS community (Siepmann et al. 2011; ChristensenJeffries et al. 2014; Ackermann and Schmitz 2016). The latter uses the bubble trajectories for reconstructing the vascular systems and performing flow measurement in micro- and macro- vessels at very high spatial resolution. The applications of ULM include mapping the cerebral and renal vascular systems in rodent models (Errico et al. 2015; Foiret et al. 2017; Lin et al. 2017), and detecting a small deep-seated human cerebral aneurysm (Demené et al. 2021). In the present study, ULM is adopted for visualizing and quantifying the cerebral microcirculation in piglet models.

Since the width of micro-vessels is comparable to the spatial resolution of the ultrasound systems, application of ULM requires precise localization and robust tracking of the bubbles. Previous localization techniques have involved deconvolution (Foroozan et al. 2018; Zhang et al. 2020), as well as correlation (Demené et al. 2021) and peak thresholding (Jeronimo et al. 2020). Recent studies have shown that preprocessing of the CEUS images using deep learning based techniques could reduce the error in bubble localization by $75 \%$ (Liu et al. 2020), and could be about 60 times faster than blind deconvolution (BD) methods (Bai et al. 2019). The robustness and generalizability of deep learning models rely on a training dataset that represents a wide range of circumstances. However, CEUS images recorded by different ultrasound systems, contrast agents, and imaging settings, as well as recoded at various depth and organs, involve diverse noise levels, backgrounds, and bubble image morphologies. These variations undermine the robustness of the previous models that are based on specific spatially invariant point spread functions (PSF) to describe the bubble image morphologies (Liu et al. 2020; van Sloun et al. 2020). Therefore, further imprvements to these models are needed for CEUS images recorded by curved, vector, and curvilinear probes, which have a PSF that becomes increasingly elongated with increasing depth and has an orientation that varies with angular position. In terms of bubble tracking, taking advantage of the ultrafast $(\sim 1000$ frames/s) ultrasound research systems, the nearest-neighbor data association strategy is common in ULM applications (Christensen-Jeffries et al. 2014; Errico et al. 2015). For low-frame-rate clinical systems ( 50 frames/s), Ackermann et al. (2016) have developed a motion model based modified Markov chain Monte Carlo framework. Moreover, a recent work has proposed a Kalman filter based tracking method (Tang et al. 2020). In the present work, the spatio-temporal consistency conditions in the flow direction are incorporated to the existing Kalman filter framework (Kim et al. 2015) to improve the bubble tracking.

In this paper we utilize echo-PTV to perform ULM for visualizing the cerebral microcirculation in pediatric pig models using a clinical CEUS system. A U-net based deep learning model is designed and trained by CEUS and synthetic images for bubble detection in images involving a wide range of PSFs. The new procedure substantially outperforms blind deconvolution based methods in terms of the processing speed and localization accuracy. The bubble tracking combines a Kalman filter, several candidate selection criteria, and globally optimized candidate assignments. The paper concludes with a sample application to visualize the cerebral microcirculation in a piglet.

\section{Methods}

\subsection{Animal preparation and CEUS imaging}

The current study utilizes a pediatric pig model (female, 4 -week-old, $10 \mathrm{~kg}$ ) to visualize and quantify the cerebral micro-perfusion. All of the animal preparation and management protocols, including anesthesia, ventilation, temperature management, cannulation, neuromonitoring, and hemodynamic monitoring, have followed previously published procedures (Friess et al. 2015), which have been approved by the Institutional Animal Care and Use Committee of the Children's Hospital of Philadelphia. The CEUS scans are performed using a Siemens ACUSON Sequoia system (Siemens Medical Solutions, Malvern, PA) with a 9EC4 
transducer (Siemens Medical Solutions, Malvern, PA). To obtain clear images, a $2.5 \mathrm{~cm}$ cranial window is drilled upper right to the midline in the parietal region with intact dura. The field of view is aligned to a coronal plane containing the maximum transverse diameter of the bilateral thalami and is focused on the left hemisphere to maintain image acquisition rate of 44 frames/s. The ultrasound probe is fixed to the experimental table using a stereotactic arm. The piglet's head is also fixed to the table to minimize its motion. The contrast agents (Lumason, Bracco Diagnostics, NJ) are infused at $0.6 \mathrm{~mL} / \mathrm{min}$ using a veterinary syringe pump (PRACTIVET, Tempe, Arizona, USA) connected to the femoral vein line. This infusion rate is selected, based on a pre-study, to establish a bubble concentration low enough to facilitate detection and tracking of individual bubbles while still maintaining a sufficient number for fully mapping the vascular structures. The ideal concentrations vary between 150 to 200 bubbles per image. The recording lasts for 2 mins with 5760 images collected. The CEUS imaging is taken at dual view mode with a CEUS image on the left and a normal B-mode image on the right. The B-mode image is used as a reference for crosscorrelation based image stabilization, if needed.

\subsection{Image preprocessing using $U$-net}

In the present study, the U-net creation, training, and validation are performed using the Deep Learning Toolbox in MATLAB. The current U-net (Ronneberger et al. 2015) image enhancement procedure is illustrated in Fig. 1. This architecture is widely-used for medical imaging processing tasks, and has been reported to deliver good performances in image enhancement, object detection, and morphometry (Falk et al. 2019). As shown in Fig. 1(a), U-net consists of encoding (down-sizing) and decoding (up-sizing) steps for converting big and blurred bubble traces with varying sizes to smaller and sharper ones. The current Unet model has an encoder-decoder depth of 3, resulting in 45 layers. The inputs, i.e., $128 \times 128$ pixels grayscale images, are downsized by half at each encoder level and doubled at each decoder level. The final convolution layer is used to gather the information from the last decoder level, followed by a regression layer, which generates the output.

The network is trained by two sets of image pairs: The first set consists of raw CEUS images (training input) and the corresponding enhanced images based on blind deconvolution (training reference). To prepare the training set, the background intensity is removed by subtracting the ensemble averaged image, and rejecting pixels with intensity falling below 0.1 ( $0-1$ range), which is considered as noise. Then the intensity range for each image is re-normalized to 0-1. A sample of the resulting image is shown in Fig. 1(b). These steps ensure standardized inputs from different experiments. The enhanced training reference is generated following a modified blind deconvolution procedure (Zhang et al. 2020). Specifically, the raw CEUS images are modelled as multiple impulse signals blurred by spatially variant PSFs with additional noise. By dividing the raw images into smaller windows, a local PSF is estimated following Pan et al. (2014) in each window. PSFs estimated from 20 random raw images are averaged at each location and used to deconvolve the rest of the images. The deconvolved ones are subsequently enhanced using a modified histogram equalization method (Roth and Katz 2001). Then the corresponding pre-processed raw \& enhanced images are zeropadded on the right and bottom, and split into $128 \times 128$ pixels sub-images with $20 \%$ lateral overlapping and 15\% vertical overlapping (yellow boxes in Fig. 1(b)). These choices ensure that at least one of the neighboring sub-images contains a complete PSF. The size of the sub-image is selected to maintain the smallest PSF larger than 2 pixels after the three encoding phases of the U-net. Splitting of the images avoids large training datasets and computational cost caused by treating the whole image as a training input. Moreover, it is more focused on the local features of the PSF without depth and angular position information, making this model more robust to the various PSF shapes and orientations.

The second training set consists of synthetic images generated based on the PSF estimated by BD (Fig. 1(c)). The purpose of including synthetic images in the training data is to enhance the U-net model with cases where the precise location and morphology information are known. To mimic the result of BD and generate the training reference, 2D gaussian signals with peak intensity of 1 and diameters of 7 to 10 pixels are randomly placed on the $128 \times 128$ pixels black background (Fig. 1(c)). The bubble densities in the synthetic data are set as $8.5,25.5$, and 42.5 bubbles $/ \mathrm{cm}^{2}$, corresponding to sparse, normal, and dense bubble distributions in the raw CEUS images. Then, the previously determined, spatially varying PSF (Fig. 1(c), left side) is used for generating the training inputs. These PSFs are further randomly rotated in the $-20^{\circ}$ to 
$+20^{\circ}$ range, resized with a factor in the 0.8 to 2 range, and given a peak intensity in $0.1-1$ range to simulate the uneven illumination of the bubbles in the actual CEUS images. Such broadening of image conditions is designed to expand the capability of the U-net model to handle varying PSF morphologies, orientations, and image qualities. A sample synthetic image pair is presented in Fig. 1(c), with the blurred image on top being the training input, and the corresponding training reference, in the bottom.

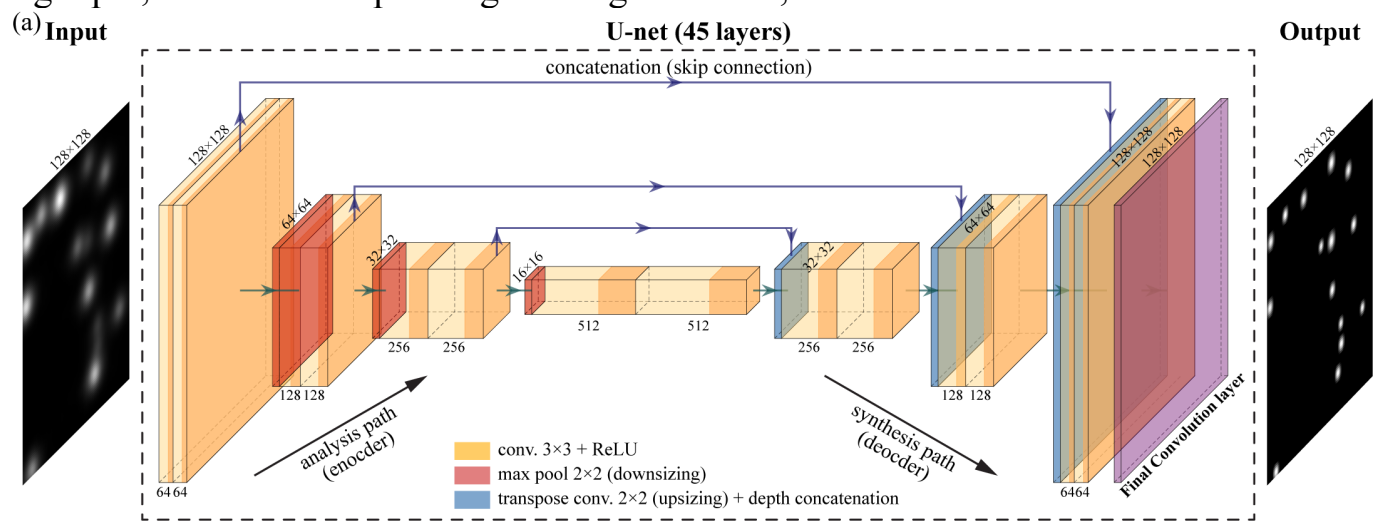

(b)

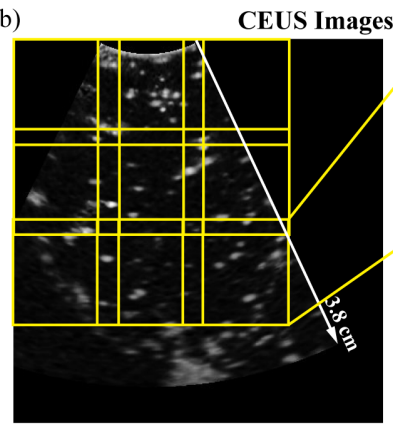
transpose conv. $2 \times 2$ (upsizing) + depth concatenation

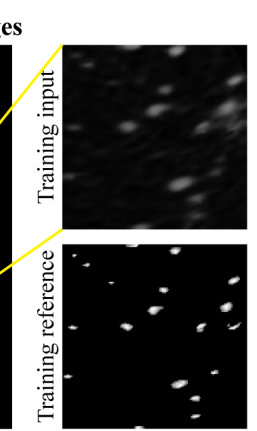

(c)

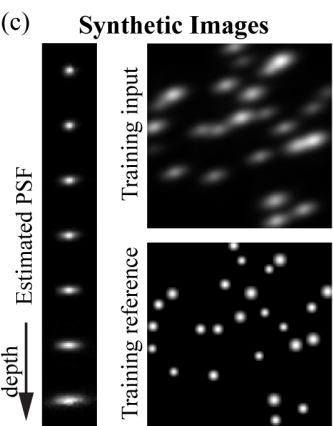

Figure 1 Schematics depicting the overall structure of the current deep learning model. (a) An illustration of the structure of the U-net with encoder depth of 3 and 45 layers in total. (b) Training data based on the actual CEUS images. The raw and enhanced images are divided into $128 \times 128$ sub-images with $20 \%$ lateral overlapping and 15\% vertical overlapping (yellow box). A sample sub-image pair of the training data is shown on the right, with the raw image (top) as training input and enhanced image (bottom) as

training reference. (c) Training data based on the synthetic images. The PSF (left column) used for generating the synthetic images are estimated by the blind deconvolution. The increasing elongation of the

PSF with depth are evident in both (b) and (c). The synthesized images at the same location in (b) is presented in the right column of (c). All raw \& enhanced images pairs (both CEUS and synthetic) are gathered and shuffled before training.

The training process is based on 400000 pairs of raw \& enhanced CEUS sub-images along with 64000 of synthetic image pairs. The training set consists of $70 \%$ of both types of images, and the rest (30\%) are used as the validation set. The Adam optimization algorithm, which is provided in the Deep Learning Toolbox of MATLAB, is used to train the model, with a total of 40 epochs. For each epoch, the dataset is randomly shuffled to increase the randomness in the training and bypass local optima. The training procedure involves a minibatch size of 64 , and initial learning rate of 0.001 . A learning rate decay strategy is used to reduce the learning rate by half when the training loss plateaus (Shin et al. 2016). During the training, the intermediate result is validated twice per epoch. The training stops when the loss does not keep decreasing for the next 5 validations. All the calculations are carried out on a PC, equipped with a NVidia GeForce RTX 2080Ti GPU (11 GB RAM), 1 Intel i9-7920X CPU (2.9 GHz), and 128 GB DDR4 RAM. Due to current amount of data and GPU RAM size, the training takes $19 \mathrm{~h}$ to converge. The final validation root-mean-squared-error for each pixel is $1.15 \times 10^{-9}$. 


\subsection{Tracking of the microbubbles}
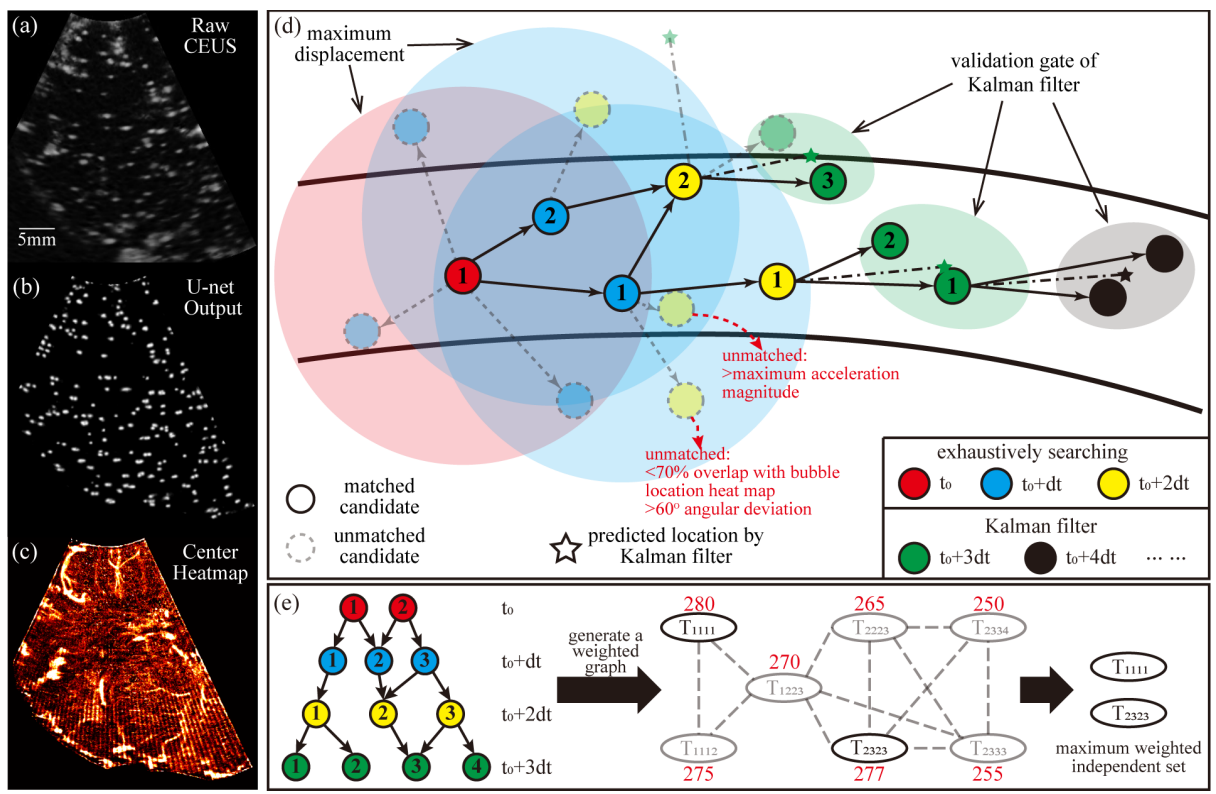

Figure 2 Schematics depicting the bubble tracking procedures. (a) A sample raw CEUS image. (b) The U-net output showing the enhanced images of (a). (c) The bubble center heatmap used as one of the references for bubble tracking. (d) An illustration of the bubble tracking strategy. For the initial 3 time steps, all candidates within a prescribed maximum displacement range are selected. Starting from the $4^{\text {th }}$ time step, the candidates are selected within the validation gate of the predicted location by Kalman filter. Several criteria, including the overlap percentage with the bubble center heatmap, continuity in

flow speed and direction, bubble morphology, and deviation from the reference flow direction, are used for removing the most unlikely candidates. (e) Global optimum data assignment. The strategy in (d) yields the same bubble being assigned to different trajectories. Here each trajectory is modeled as a node of an undirected graph, the tracking score from Kalman filter as the weight of each node, and two nodes are linked if they share the same bubble. The maximum weight independent set of this graph is the global optimized assignment of the bubbles.

Due to the complexity of the microvasculature, the following procedure has been used for bubble tracking. The raw CEUS images (Fig. 2(a)) are enhanced using the U-net described above to obtain the image shown in Fig. 2(b). Here, the elongated bubble traces are replaced by sharp images where adjacent bubbles are sufficiently separated. Subsequently, intensity-weighted center of each bubble is used for generating a heatmap of bubble locations (Fig. 2(c)), which restricts the likely locations of blood vessels during the bubble tracking process. The procedures for bubble tracking are illustrated in Fig. 2(d). The trajectories are initialized by exhaustively searching for candidates within a prescribed maximum displacement range for the first three exposures (Fig. 2(d)). The corresponding candidates in the fourth and subsequent exposures are updated using a Kalman filter (Kim et al. 2015). Removal of the most unlikely candidate tracks is based on a minimum Kalman filter tracking score, overlap with the heatmap ( $<90 \%$ for major vessels and $<50 \%$ for other regions), deviations in bubble radius ( $>50 \%)$ and directions of velocity $\left(>60^{\circ}\right)$, as well as magnitude of acceleration ( $>30 \%$ of prescribed maximum displacement). These thresholds are selected based on previous experiences (Zhang et al. 2020), and examination of numerous images. Considering that in some cases the same bubble is assigned to multiple tracks, a data association procedure is used for determining the global optimal bubble assignment (Fig. 2(e)). This step involves: i) generating an undirected weighted graph by assigning each possible trajectory to a node, assigning a weight to each node based on its tracking score representing the likelihood of being the correct trajectory, and linking nodes that share the same bubble; and ii) solving for the maximum weighted independent set (Papageorgiou and Salpukas 2009) of this graph. The latter is the largest subset of the nodes that are not interconnected and have the maximum 
average weight. Therefore, the outcome of this method gives the globally optimized candidate-trajectory association. Next, a reference flow direction map is generated after processing the first $25 \%$ of images. Then, the whole analysis is initiated again, with an additional criterion limiting the deviation in flow direction to less than $15^{\circ}$ from that of the reference. Finally, the vascular structures are visualized by super-positioning all the trajectories consisting of at least 4 exposures. The corresponding velocity map is generated by averaging the bubble velocities at each pixel.

\section{Result \& Discussion}
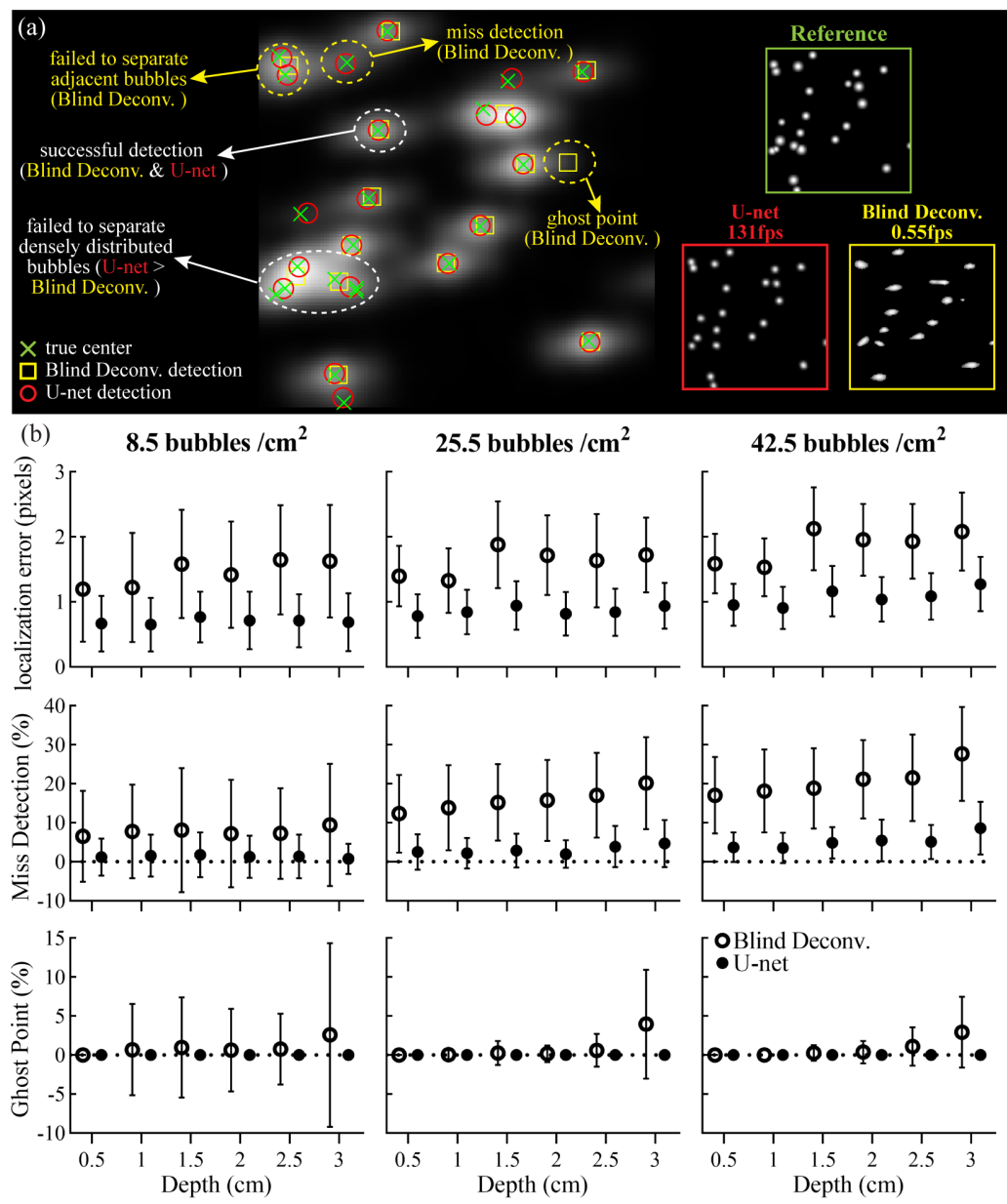

Figure 3 Evaluation and comparison of the U-net results with BD. (a) An illustration of the image enhancement and bubble localization result of U-net (red) and BD (yellow). Several issues regarding bubble detection including miss detection, generation of ghost points, and failure to separate adjacent bubbles are noted. (b) Comparisons of the localization error, percentage of miss detections and ghost points between U-net and BD at different depth and bubble densities.

\subsection{Evaluation of the U-net}

To evaluate the performance of the U-net model, 900 synthetic images pairs are generated following the procedures described in section 2.2 and processed using both U-net and BD methods. Comparisons between the two methods are presented in Fig. 3. Fig. 3(a) a visualization of the synthetic reference images (green) and the outputs of both the U-net (red) and BD (yellow). The images are shown separately on the right side, and superimposed on the synthetic blurred images on the left. It is evident that the U-net result is much sharper and closer to the reference than the BD based result. The processing speed of U-net is 131 frames/s using a GPU, while that of BD is 0.55 frames/s using 12 core CPU. A GPU based BD code is not available. 
Further acceleration of the U-net model could be easily achieved by expanding the RAM of the GPU. Several issues are listed regarding the bubble detection on the left panel of Fig. 3(a). While both techniques detect most of the bubbles, the BD method is more prone to miss detections than the U-net. Moreover, a ghost point, i.e., erroneous detection caused by enhancement of the pixels which do not belong to a real bubble, has been generated by the BD method. Furthermore, while both methods fail to completely separate densely packed bubbles, U-net evidently outperforms BD. Quantitative analysis of these observations is presented in Fig. 3(b) for varying bubble densities (columns), and image depths, i.e., various sizes of the PSF (see Fig. 2(c)). Here, a successful detection is defined as the closest detection to a prescribed center as long as it is smaller than 6 pixels. Miss detections are the prescribed centers that cannot find a successful match, and ghost points are detections without a prescribed center in the 6 pixel range. Therefore, for three adjacent bubbles being identified by the BD method as one bubble, the result would be two misses and one successful detection. As the first row of Fig. 3(b) shows, for all bubble densities and depths, the mean and standard deviations of BD's localization error are much higher than those of the U-net. As expected, the error in BD increases with depth, while that of the U-net remains at the similar level for all depths. For both methods, the error increases with bubble density. However, for low $\left(8.5 \mathrm{bubbles} / \mathrm{cm}^{2}\right)$ and normal $(25.5$ bubbles $/ \mathrm{cm}^{2}$ ) densities, the errors for U-net are less than 1 pixel. Furthermore, BD also has more miss detections than U-net (second row of Fig. 3(b)). While the average miss detections for U-net are typically less than 5\%, those of BD not only increase with depth, but also increase significantly with bubble density. As for the ghost points ( $3^{\text {rd }}$ row in Fig. 3(b)), U-net is not prone to have such an issue for all the tested depths and densities, and BD shows about $5 \%$ of ghost points at large depth. Clearly, U-net outperforms BD in terms of both processing speed and accuracy in enhancing CEUS images and detecting microbubbles.
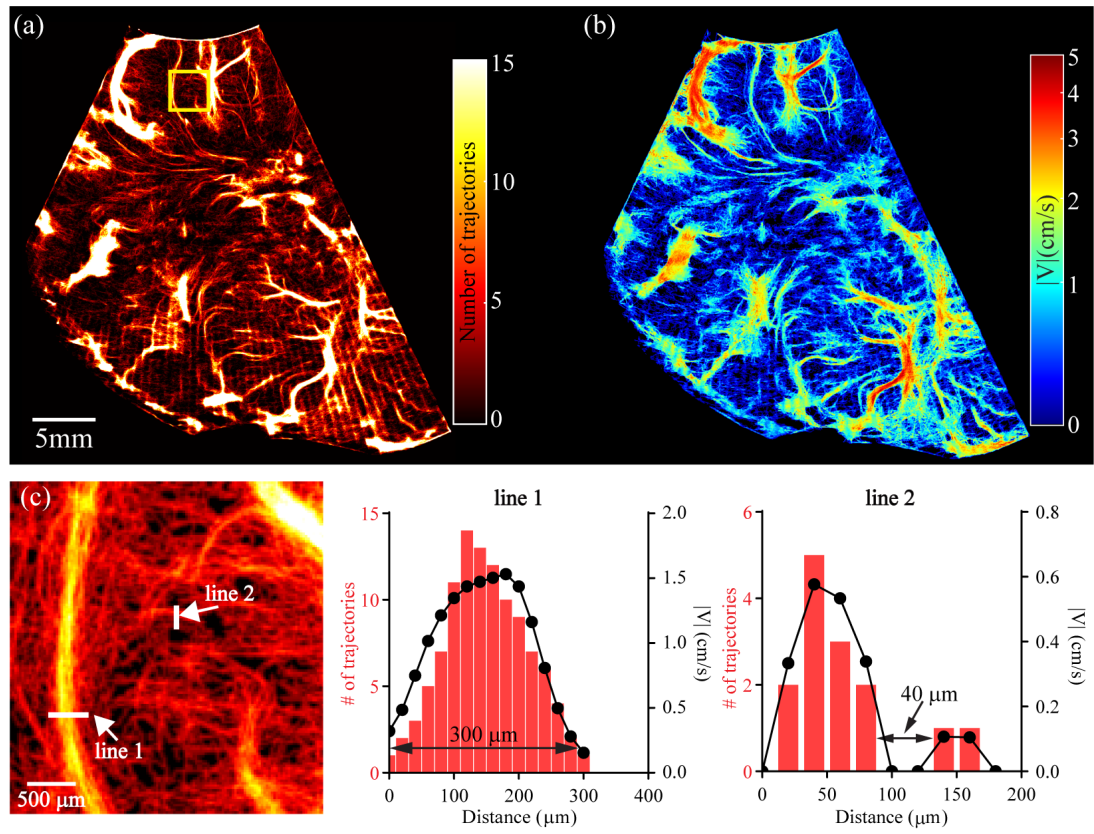

Figure 4 Visualization and quantification of the cerebral microcirculation. (a) A visualization of the blood vasculatures. (b) The corresponding blood velocity map of (a). (c) A magnified view of the region in the yellow box of (a), showing the complex microvascular networks. Line 1 is the cross section (red bar plot) of a moderate blood vessel, and the result for line 2 shows that the current work is able to distinguish micro-vessels departed by $40 \mu \mathrm{m}$.

\subsection{Visualization and quantification of the cerebral microcirculation}

The visualization of the vasculature is presented in Fig. 4(a), and the corresponding time-averaged velocity map is demonstrated in the Fig. 4(b). Major blood vessels ( $\sim 1 \mathrm{~mm}$ width) with time-averaged speeds of more than $5 \mathrm{~cm} / \mathrm{s}$ and moderate vessel $(\sim 300 \mu \mathrm{m}$ width $)$ with speeds in $1-2 \mathrm{~cm} / \mathrm{s}$ range are evident. 
$14^{\text {th }}$ International Symposium on Particle Image Velocimetry -- ISPIV 2021

August 1--5, 2021

The speeds for micro-vessels are usually below $1 \mathrm{~cm} / \mathrm{s}$. A closer look at the micro-vessel networks is presented in Fig. 4(c), where a magnified view of the region in the yellow box of Fig. 4(a) is demonstrated. Two lines are indicated on the zoomed view to demonstrate the spatial resolution of the current result. Line 1 is the cross section of a moderate sized blood vessel, and the bar plot of the numbers of trajectories along this line shows that the in-plane width of this vessel is around $300 \mu \mathrm{m}$. The corresponding velocity profile shows a parabolic shape, which is consistent with the expected Poiseuille flow profile. Line 2 is placed across two parallel micro-vessels that are very close to each other. The results of line 2 shows that the spatial resolution of the current method is sufficient to distinguish two neighboring micro-vessels that are $40 \mu \mathrm{m}$ apart.

\section{Conclusions}

In conclusion, the current work has proposed a U-net based image processing method and a Kalman filter based bubble tracking method for performing echo-PTV using clinically available CEUS images. The Unet model has significantly improved the processing speed and accuracy in bubble detection. We have demonstrated the application of the currently proposed method for measuring the cerebral microcirculation on a piglet. Neighboring micro-vessels that are $40 \mu \mathrm{m}$ apart from each other can be successfully detected.

\section{Acknowledgements}

Misun Hwang acknowledges support from NIH grants R01 NS119473-01, and Joseph Katz acknowledges support from the Department of Mechanical Engineering at Johns Hopkins University.

\section{References}

Ackermann D, Schmitz G (2016) Detection and Tracking of Multiple Microbubbles in Ultrasound B-Mode Images. IEEE Transactions on Ultrasonics, Ferroelectrics, and Frequency Control 63:72-82

Bai C, Liu C, Yu X, Peng T, Min J, Yan S, Dan D, Yao B (2019) Imaging enhancement of light-sheet fluorescence microscopy via deep learning. IEEE Photonics Technology Letters 31:1803-1806

Christensen-Jeffries K, Browning RJ, Tang M-X, Dunsby C, Eckersley RJ (2014) In vivo acoustic superresolution and super-resolved velocity mapping using microbubbles. IEEE transactions on medical imaging 34:433-440

Crapper M, Bruce T, Gouble C (2000) Flow field visualization of sediment-laden flow using ultrasonic imaging. Dynamics of Atmospheres and Oceans 31:233-245

Dankbaar JW, De Rooij NK, Rijsdijk M, Velthuis BK, Frijns CJM, Rinkel GJE, Van Der Schaaf IC (2010) Diagnostic threshold values of cerebral perfusion measured with computed tomography for delayed cerebral ischemia after aneurysmal subarachnoid hemorrhage. Stroke 41:1927-1932

Demené C, Robin J, Dizeux A, Heiles B, Pernot M, Tanter M, Perren F (2021) Transcranial ultrafast ultrasound localization microscopy of brain vasculature in patients. Nature Biomedical Engineering $5: 219-228$

Errico C, Pierre J, Pezet S, Desailly Y, Lenkei Z, Couture O, Tanter M (2015) Ultrafast ultrasound localization microscopy for deep super-resolution vascular imaging. Nature 527:499-502

Falk T, Mai D, Bensch R, Çiçek Ö, Abdulkadir A, Marrakchi Y, Böhm A, Deubner J, Jäckel Z, Seiwald K (2019) U-Net: deep learning for cell counting, detection, and morphometry. Nature methods 16:67-70

Foiret J, Zhang H, Ilovitsh T, Mahakian L, Tam S, Ferrara KW (2017) Ultrasound localization microscopy to image and assess microvasculature in a rat kidney. Scientific reports 7:1-12 
$14^{\text {th }}$ International Symposium on Particle Image Velocimetry -- ISPIV 2021

August 1--5, 2021

Foroozan F, O'Reilly MA, Hynynen K (2018) Microbubble localization for three-dimensional superresolution ultrasound imaging using curve fitting and deconvolution methods. IEEE Transactions on Biomedical Engineering 65:2692-2703

Friess SH, Bruins B, Kilbaugh TJ, Smith C, Margulies SS (2015) Differing effects when using phenylephrine and norepinephrine to augment cerebral blood flow after traumatic brain injury in the immature brain. Journal of neurotrauma 32:237-243

Hanlo PW, Gooskens R, Nijhuis IJM, Faber JAJ, Peters RJA, Van Huffelen AC, Tulleken CAF, Willemse J (1995) Value of transcranial Doppler indices in predicting raised ICP in infantile hydrocephalus. Child's Nervous System 11:595-603

Hwang M (2019) Introduction to contrast-enhanced ultrasound of the brain in neonates and infants: current understanding and future potential. Pediatric radiology 49:254-262

Jeronimo MD, Najjari MR, Rival DE (2020) Echo-Lagrangian particle tracking: an ultrasound-based method for extracting path-dependent flow quantities. Measurement Science and Technology 31:54008

Kim C, Li F, Ciptadi A, Rehg JM (2015) Multiple hypothesis tracking revisited. In: Proceedings of the IEEE international conference on computer vision. pp 4696-4704

Kim H-B, Hertzberg JR, Shandas R (2004) Development and validation of echo PIV. Experiments in fluids $36: 455-462$

Leliefeld PH, Gooskens RHJMJM, Vincken KL, Ramos LMPP, van der Grond J, Tulleken CAFF, Kappelle LJ, Hanlo PW (2008) Magnetic resonance imaging for quantitative flow measurement in infants with hydrocephalus: a prospective study. Journal of Neurosurgery: Pediatrics 2:163-170

Lin F, Shelton SE, Espíndola D, Rojas JD, Pinton G, Dayton PA (2017) 3-D ultrasound localization microscopy for identifying microvascular morphology features of tumor angiogenesis at a resolution beyond the diffraction limit of conventional ultrasound. Theranostics 7:196

Liu X, Zhou T, Lu M, Yang Y, He Q, Luo J (2020) Deep learning for ultrasound localization microscopy. IEEE transactions on medical imaging 39:3064-3078

Pan J, Hu Z, Su Z, Yang M-H (2014) Deblurring text images via L0-regularized intensity and gradient prior. In: Proceedings of the IEEE Conference on Computer Vision and Pattern Recognition. Columbus, OH, USA, pp 2901-2908

Papageorgiou DJ, Salpukas MR (2009) The maximum weight independent set problem for data association in multiple hypothesis tracking. In: Optimization and Cooperative Control Strategies. Springer, pp 235255

Poelma C (2017) Ultrasound imaging velocimetry: a review. Experiments in Fluids 58:1-28

Ronneberger O, Fischer P, Brox T (2015) U-Net: Convolutional Networks for Biomedical Image Segmentation BT - Medical Image Computing and Computer-Assisted Intervention - MICCAI 2015. In: Navab N, Hornegger J, Wells WM, Frangi AF (eds). Springer International Publishing, Cham, pp 234241

Roth GI, Katz J (2001) Five techniques for increasing the speed and accuracy of PIV interrogation. Measurement Science and Technology 12:238

Sampath K, Harfi TT, George RT, Katz J (2018) Optimized time-resolved echo particle image velocimetryparticle tracking velocimetry measurements elucidate blood flow in patients with left ventricular thrombus. Journal of biomechanical engineering 140:41010

Shin H-C, Roth HR, Gao M, Lu L, Xu Z, Nogues I, Yao J, Mollura D, Summers RM (2016) Deep convolutional neural networks for computer-aided detection: CNN architectures, dataset characteristics 
$14^{\text {th }}$ International Symposium on Particle Image Velocimetry -- ISPIV 2021

August 1--5, 2021

and transfer learning. IEEE transactions on medical imaging 35:1285-1298

Siepmann M, Schmitz G, Bzyl J, Palmowski M, Kiessling F (2011) Imaging tumor vascularity by tracing single microbubbles. In: 2011 IEEE International Ultrasonics Symposium. IEEE, pp 1906-1909

Tang S, Song P, Trzasko JD, Lowerison M, Huang C, Gong P, Lok U-W, Manduca A, Chen S (2020) Kalman Filter-Based Microbubble Tracking for Robust Super-Resolution Ultrasound Microvessel Imaging. IEEE Transactions on Ultrasonics, Ferroelectrics, and Frequency Control 67:1738-1751

van Sloun RJG, Solomon O, Bruce M, Khaing ZZ, Wijkstra H, Eldar YC, Mischi M (2020) Super-resolution ultrasound localization microscopy through deep learning. IEEE Transactions on Medical Imaging 40:829-839

Zaharchuk G, Mandeville JB, Bogdanov AA, Weissleder R, Rosen BR, Marota JJ (1999) Cerebrovascular dynamics of autoregulation and hypoperfusion. An MRI study of CBF and changes in total and microvascular cerebral blood volume during hemorrhagic hypotension. Stroke 30:2195-2197

Zhang Z, Zhou X, Suarez-Pierre A, Lui C, Kearney S, Yeung E, Halperin H, Choi CW, Katz J (2020) TimeResolved Echo-Particle Image/Tracking Velocimetry Measurement of Interactions Between Native Cardiac Output and Veno-Arterial ECMO Flows. Journal of Biomechanical Engineering 143: 